\title{
Incorporación de las tecnologías de información y comunicación (TIC) durante el proceso de aprendizaje en la educación superior
}

\author{
Derly F. Poveda-Pineda* y José E. Cifuentes-Medina \\ Universidad Pedagógica y Tecnológica de Colombia, Facultad de Estudios a Distancia, Escuela de Ciencias \\ Humanísticas y Educación, Tunja, Colombia. (correo-e: derly.poveda@uptc.edu.co; joseeriberto.cifuentes@uptc.edu.co)
}

* Autor a quien debe ser dirigida la correspondencia.

Recibido Mar. 27, 2020; Aceptado May. 27, 2020; Versión final Jul. 10, 2020, Publicado Dic. 2020

\section{Resumen}

En este estudio se aborda la incorporación de la tecnología en la medicación pedagógica y su influencia en el desarrollo de la educación superior. Se describe un sustento teórico cuyos postulados muestran la incidencia de las tecnologías de información y comunicación (TIC) en el aprendizaje en la educación superior. El método es cuantitativo y la investigación es realizada en el programa de licenciatura en educación básica de la Universidad Pedagógica y Tecnológica de Colombia. Los resultados muestran la importancia de la utilización y adaptación de la tecnología al servicio de estilos de aprendizaje diversos y sugieren que ésta por sí sola no constituye un factor de innovación. Se concluye que las TIC cobran un rol importante para el desarrollo de las estrategias docentes en el programa de licenciatura en educación básica y que del rol que asuman los estudiantes depende el éxito del ejercicio académico.

\section{Incorporation of information and communication technologies (ICT) during the learning process in higher education}

\begin{abstract}
The present study examines technology incorporation in pedagogical mediation and its influence on the development of higher education. A theoretical support is described with detailed postulates that present the incidence of information and communication technologies (ICT) in higher education learning. The method is qualitative and the research is conducted in the basic education bachelor's program at the Pedagogical and Technological University of Colombia. The results show the importance of using and adapting technology to diverse learning modes and suggest that technology on its own is not an innovation factor. It is concluded that ICTs acquire an important role for the development of teaching strategies in the basic education bachelor's program and that academic success depends on the roles that students assume.
\end{abstract}

Keywords: learning; higher education; technology; quality educational model 


\section{INTRODUCCIÓN}

El uso de las TIC en los procesos de aprendizaje, ha cobrado importancia en la educación superior debido a los cambios metodológicos y los retos que estas herramientas traen consigo para enfrentar otras formas de comunicación y acceso al conocimiento, en las universidades. La articulación entre los procesos de aprendizaje y la aparición de la tecnología, se constituyen en elementos transformadores de la metodología de enseñanza, en la cual se atiende con especial atención a las ventajas, oportunidades y limitaciones que las TIC traen al ejercicio mismo de la docencia y al proceso de aprendizaje de los estudiantes del programa de Licenciatura en Educación Básica de la Universidad Pedagógica y Tecnológica de Colombia.

Siendo evidente la incorporación de la tecnología en la educación, se hace importante establecer el impacto de estas herramientas de mediación en la calidad educativa, el alcance de los objetivos de aprendizaje y los contenidos propuestos desde cada plan de estudios, llevando de la mano estrategias que transforman la tradicionalidad de la educación a distancia. Así, se replantean los roles de los actores del proceso educativo, donde el profesor se convierte en un guía y dinamizador de escenarios que facilita la interacción, y utiliza la tecnología para establecer relación entre el contenido y el estudiante, por eso más allá del mero componente técnico, se establecen las Tecnologías de la Relación, la Información y la Comunicación (TRIC), las cuales deben permitir validar el proceso mismo de formación, haciendo una revisión de los instrumentos utilizados, la correspondencia entre lo que se planeó y lo que se ejecutó, además de la pertinencia del sistema de evaluación desarrollada.

Es así como, la presencia de la tecnología en el proceso de aprendizaje, de los estudiantes de Licenciatura en Básica de la UPTC, ha generado un cambio en el desarrollo del ejercicio docente, trasformando significativamente las metodologías de enseñanza, el modelo de comunicación, el acceso a la información, el diseño y el contenido de los materiales de estudio. Se percibe un mejoramiento en la calidad educativa, cambios profundos en las herramientas de mediación ya que aparecen elementos como encuentros asincrónicos, mediante el uso de LMS y classroom. Los procesos de aprendizaje a partir de la utilización de nuevas herramientas de comunicación, rompen las barreras de espacio y tiempo, y constituyen oportunidades para construir comunidades académicas, a partir de la indagación y el análisis que configuran el conocimiento con la realidad del contexto donde se aprende.

La incorporación de las TIC en la educación superior, hoy es una realidad, ahora el reto transciende a la combinación de metodologías de aprendizaje que fomente en el rol docente, la vinculación de estrategias pedagógicas apoyadas la gamificación, el aprovechamiento de herramientas digitales en línea y el uso adecuado de las TRIC para responder con las exigencias educativas actuales. De esta forma, se hace importante determinar la incidencia de la incursión de la tecnología en los procesos educativos de los Licenciados en Básica de la UPTC, en los cuales se hace presente el uso masivo de las TIC para la interacción; se ajustan tiempos y contenido curricular, además de establecer diferentes estrategias pedagógicas en las cuales el protagonismo de los estudiantes en su proceso de formación se hace cada vez más evidente y determinante para el mejoramiento y el éxito del proceso que se desarrolla.

Las TIC están ofreciendo a los estudiantes acceso ilimitado a fuentes de conocimiento, a herramientas multimedia y contenidos basados en la gamificación. Además, las TRIC han transformado el entorno de aprendizaje actual, pasando de uno tradicional centrado en el docente a uno centrado en el alumno (Sánchez, Añorve y Alarcón, 2017). El interés por fomentar el uso de la tecnología en la UPTC, está marcado por la necesidad de responder a las políticas académicas y a las exigencias de una formación integral en la cuales el egresado de las licenciaturas responsan con la calidad y el profesionalismo que marcó el siglo XXI al modernizar las sociedades y aligerar los niveles de vida (Remache y Belarbi, 2019), que se enmarca en el fomento del desarrollo económico y cultural del país.

Aunque se espera en muchos escenarios educativos que la presencia de la tecnología sea una solución automática para que las metodologías de aprendizaje sean más efectivas, por cuanto sustituyen la tradicionalidad de la enseñanza, para el caso de la UPTC, el modelo de formación de los licenciados necesitó de una transición progresiva, dando inicio con el uso de Moodle como principal sistema de gestión para administrar el aprendizaje; y la formación del profesorado en el diseño de situaciones pedagógicas que llevarán al contraste con la realidad y la praxis de la labor de los futuros egresados. Las TRIC traen ventajas importantes para facilitar la interacción con el aprendizaje y alcanzar los objetivos de formación mediante estrategias diferentes para su desarrollo, es uno de los aspectos a resaltar durante la investigación, además de la percepción que tiene la comunidad de la UPTC con relación al tema.

El objetivo principal del estudio se enmarcó en determinar los retos y oportunidades de incorporar las TIC en el Programa de la Licenciatura en Educación Básica de la UPTC; para lo cual se definieron algunos objetivos específicos como la búsqueda de sustentos teóricos para identificar el rol que ha desempeñado la tecnología en la educación superior, el análisis de la percepción de los actores del proceso educativo frente al uso de la 
tecnología en el modelo educativo de formación de licenciados; analizar la influencia del uso de las TIC en la calidad de la formación recibida y finalmente concluir de los alcances y las prácticas desarrolladas en la UPTC, para incorporar de manera exitosa las TIC en el modelo pedagógico de la educación a distancia, enmarcado en la tradicionalidad.

El estudio se registra en la línea de investigación, Educación a distancia y virtual, del Doctorado en Educación. El análisis frente a la pertinencia de la utilización de la tecnología dentro del proceso de aprendizaje de los Licenciados de Básica en la UPTC, toma en cuenta los retos y las oportunidades que estas herramientas traen en las prácticas educativas; las cuales han establecido para su desarrollo, un ejercicio mediador y facilitador de escenarios para favorecer el aprendizaje y así alcanzar los objetivos planteados al interior de los programas académicos en las universidades. Se describe un apartado de generalidades, el aprendizaje mediado por las TIC; además se señala la metodología llevada a cabo, los resultados y las conclusiones obtenidas del proceso desarrollado. Determinar los retos y oportunidades de incorporar las TIC en el Programa de la Licenciatura en Educación Básica de la UPTC, es uno de los mayores desafíos alcanzados con el estudio que se describe.

\section{LAS TIC EN LA EDUCACIÓN SUPERIOR}

La presencia de la tecnología en las aulas de clases, es hoy una realidad de la educación superior; sin embargo; los instrumentos tecnológicos por sí solos no van a mejorar el trabajo dentro del aula; se requiere un esfuerzo mayor que considere aspectos formativos y de contenido que involucren a los participantes (Ortega y Casillas, 2014); así el cambio de metodologías para la enseñanza y el aprendizaje al interior de las universidades, ha sido progresivo y acelerado; para de esta forma afrontar las exigencias de la competitividad que la sociedad del conocimiento exige. A casi dos décadas de la Conferencia Mundial sobre Educación Superior en el Siglo XXI, en cuyas declaraciones se vislumbraba el surgimiento de la sociedad fundada en el conocimiento, de su acompañamiento de las TIC y el papel que desempeñaría la educación en esta transición social, los actores educativos seguimos explorando las funciones, implicaciones y consecuencias de su uso (Herrera, 2015). Esto, trae consigo la necesidad de evaluar la incidencia del uso de estas herramientas en la formación de profesionales y sus implicaciones en la vida laboral; siendo uno de los principales retos de la Licenciatura en Educación Básica de la UPTC, por cuanto se considera que sus beneficios redundan en la calidad educativa no solo de sus egresados, sino de las futuras generaciones que hacen parte de sus prácticas pedagógicas.

Todos los indicadores apuntan en la dirección de una incorporación creciente de las TIC al currículo y no hay razón para pensar que el manejo y dominio de estas tecnologías vaya a presentar mayores dificultades en otros contenidos curriculares (Coll, 2008). Por tanto, la incorporación de la tecnología para el desarrollo de procesos de aprendizaje en el ámbito universitario, ha cobrado mucha importancia en los últimos años y se considera que las TIC, son cada vez más amigables, accesibles y adaptables a la acción del tutor. De allí que tanto en el docente como en la institución educativa las pueden incorporar para generar mejoras en la acción didáctica; de ese modo, se puede transitar de la enseñanza tradicional hacia un aprendizaje más colaborativo y en red (Mendoza, Burbano y Valdivieso, 2018). Los maestros siempre deben estar listos y bien equipados en términos de competencias en TIC y actitud positiva para proporcionar oportunidades de aprendizaje basadas en las TIC para que los estudiantes mejoren su calidad de aprendizaje (Ghavifekr, 2014). El uso de las Tecnologías de la Información y de la Comunicación está creciendo a un ritmo acelerado y está dando lugar a cambios importantes en la sociedad, permitiendo que la información esté al alcance de todos y ofreciendo nuevos canales de comunicación y de entretenimiento (Garrote, Jiménez y Gómez, 2018).

Las experiencias educativas mediadas por las TIC, permiten visualizar el importante papel que desempeñan en las instituciones de educación superior (Figura 1), no solo en la formación de futuros profesionales haciendo énfasis en las habilidades digitales necesarias para su desarrollo en del siglo XXI, pero también como espacios adecuados para el desarrollo y la formación continua de docentes con estrategias metodológicas innovadoras que facilitan y mejoran el proceso educativo (Aranguiz y Quintana, 2016). Ahora bien, siendo la UPTC la única universidad pública en el Departamento de Boyacá (Colombia), debe afrontar con responsabilidad el reto de mantener la calidad educativa y fomentar el uso de la tecnología en todos los procesos que implique, de tal modo que sus beneficios sean un potencial de aprovechamiento para el campo de la ciencia y la investigación, dado que la actual situación por la que atraviesa el mundo, en donde azotados por la pandemia de Covid19, la educación ha dado un giro extraordinario, reconfigurando sus procesos e interacciones educativas, haciendo posible continuar con la formación de profesionales desde un modelo remoto de aprendizaje mediado por tecnologías. Se han tenido que reinventar las prácticas educativas, el rol del profesorado ha sido repensado y la necesidad de mantener la academia abierta e incluyente, mientras los edificios se encuentran cerrados en uno de los mayores desafíos que la educación superior ha enfrentado en los últimos tiempos. 


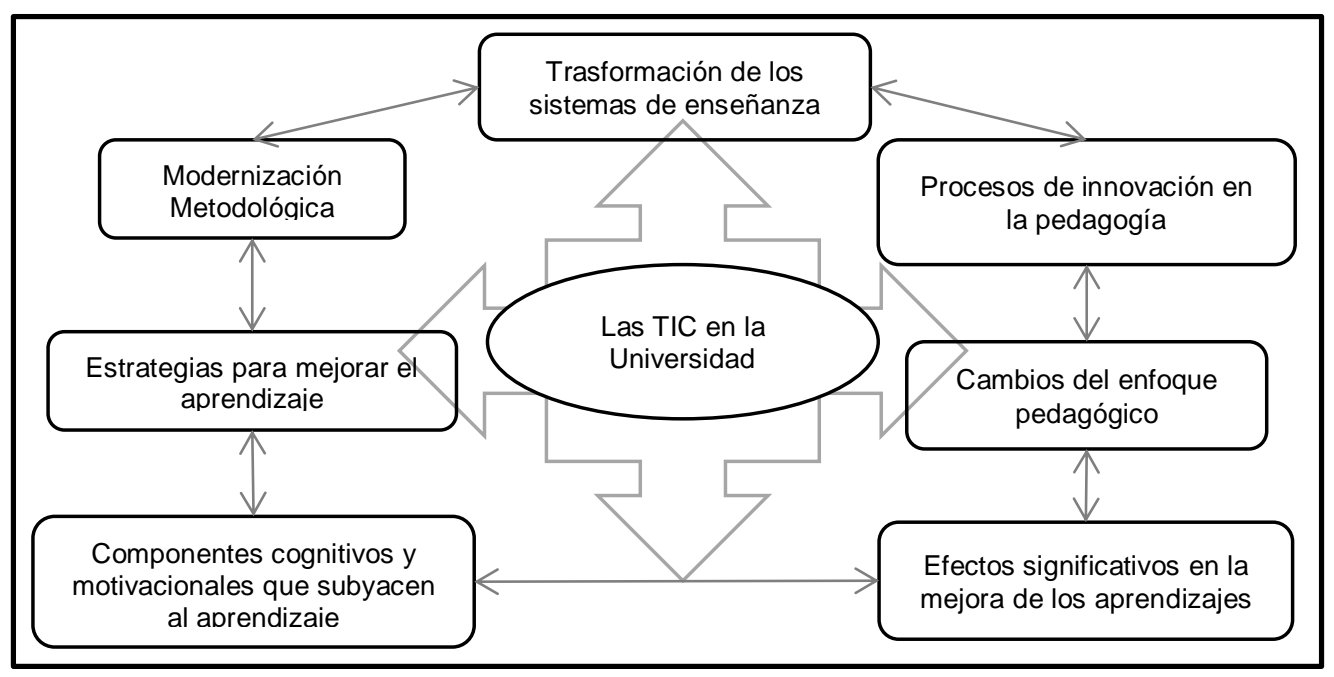

Fig.1: Las TIC en los procesos de educación superior

En consecuencia, se tiene que el estado actual del aprovechamiento de las TIC en los procesos de aprendizaje de la educación superior, al interior de la universidad, está determinada por el potencial de su utilización en pro del desarrollo de la formación y cumplimiento de los objetivos educativos. Así, están orientados a mejorar la calidad de vida de las personas dentro de un entorno y como tales se integran a un sistema de información interconectada y complementaria, lo cual permite, hoy, romper las barreras que existen entre cada uno de ellos (Colina,2008); por tanto corresponde a la educación superior abrir espacios acordes con el desarrollo tecnológico actual, en donde el b-learning, las flipped calssroom, la gamificación y otros elementos utilizados para apoyar el desarrollo del ejercicio educativo, sean evaluados, es decir, se valore la incidencia y los efectos que su uso permanente pueda generar en la calidad de los aprendizajes impartidos con el modelo de enseñanza utilizado.

La educación superior, ha sido objeto de muchas críticas e innumerables comentarios, que hoy en época de Covid19, se argumentan por los esquemas tradicionales de enseñanza, que por miedo no han dejado visibilizar el potencial metodológico que tiene el uso de las TIC en la UPTC, como herramientas que favorezcan el desarrollo que nuevos aprendizajes en los futuros licenciados de Educación Básica; ahora, se observa una oportunidad de cambio para la población naciente en este nuevo mundo tecnológico, al igual que para la población que ha crecido en una brecha tecnológica gigantesca; es la oportunidad de actualizarse e incorpore a las actuales exigencias que debe afrontar la educación.

Junto con las nuevas herramientas pedagógicas, surgieron también unas nuevas técnicas, métodos y estrategias entre los docentes y la parte organizacional de las instituciones educativas, como el mejoramiento de las comunicaciones a nivel interno, el proceso de adaptación de los contenidos académicos de cada una de las asignaturas o competencias para introducirlas en el entorno virtual (Martínez, Steffens, Ojeda y Hernández, 2018) y aunque existen barreras para el aprendizaje electrónico que incluyen clases de gran tamaño, ancho de banda limitado, tiempo y limitaciones financieras; la integración de la tecnología continúa, y los estudiantes están entusiasmados en participar en actividades (Kinuthia y Dagada, 2008). El fortalecimiento de las competencias TRIC en los docentes y generar espacios de cambios profundos en los procesos de enseñanza y aprendizaje para dar protagonismo a las prácticas mediadas por la tecnología al interior de la UPTC; no se ha hecho esperar. El autoaprendizaje y la colaboratividad, han sido factores determinantes para que el proceso tenga éxito a través de la mediación digital. Se ha volcado el interés por fomentar la educación virtual bajo nuevos modelos pedagógicos que estén mediados por los recursos de tecnologías para la comunicación y la información Facundo (2002). Sin embargo, este modelo educativo deben estar enfocados en la articulación de la pedagogía y la didáctica, mediante estrategias que fomenten el desarrollo integral del estudiante de manera tal, que se motive a interactuar en torno a la disciplina de aprendizaje al mismo tiempo que se reflexione frente a su desarrollo profesional como persona partícipe de una sociedad en constante cambio.

No es tan simple como llevar la tecnología a la educación, sino transformar el modelo educativo en donde la tecnología esté presente. El rápido desarrollo de las tecnologías, ofrece nuevas posibilidades y desafíos para la enseñanza y el aprendizaje en instituciones de educación superior. Uno de estos desafíos es la competencia de los docentes en el uso de tecnologías en desarrollo (Sointu, Valtonen, Hirsto, Kankaanpää, Saarelainen, Mäkitalo, y Manninen, 2019), la labor docente cobra otro rol y el ejercicio se hace distinto al tradicional. La resignificación de los procesos de enseñanza que llevan a pensar en la necesidad de evaluar la calidad de la actividad desarrollada al interior de la Universidad, el replanteamiento de un modelo educativo 
activo y participativo en el cual todos los esfuerzos se enmarcan en la búsqueda de diferentes formas de construir conocimiento a través de la potenciación del uso de TRIC y sistemas telemáticos que reformulan la educación del siglo XXI, hoy marcado en la historia como la época del virus, Covid19, hizo cerrar puertas de madera pero no las aulas para continuar aprendiendo.

Frente a la importancia de incluir a las TIC en los procesos de aprendizaje de la educación superior, Silvio (2000), en uno de los apartes del su libro, en los que compendia la investigación realizada para identificar como sería la transformación de la educación superior con la presencia de la tecnología; afirma que, el desarrollo de las tecnologías, que han sido denominadas TIC, es de particular significación para la educación superior, porque ella se caracteriza por ser un sistema en el cual se administran exclusivamente datos, informaciones y conocimientos. Dicho de otra forma, las TIC ridiculizaron el cambio en la educación, especialmente en la educación superior. Desde la admisión hasta los resultados, cada etapa de las TIC desempeña un papel muy vital. Las formas tradicionales de enseñanza y aprendizaje se están convirtiendo cada vez más en entornos virtuales y en línea. Existen infinitas posibilidades con la integración de las TIC en el sistema educativo. El uso de las TIC en la educación no solo mejora el proceso de aprendizaje de la enseñanza en el aula, sino que también proporciona la facilidad de e-learning. (Dave, 2019). Por tanto, son enormes las ventajas y los retos que trae a las universidades el uso de las TIC en sus modelos formativos, aunque ellas son las mismas que han llamado a mantener la estructura tradicional de aprendizaje, so pena de proteger la identidad institucional y la calidad del servicio educativo.

De igual forma, la educación superior deberá desenvolverse en el futuro en una sociedad que se ha convenido en llamar sociedad del conocimiento, en la cual el conocimiento será la fuente principal de producción, riqueza y poder (Silvio, 2000); en tanto, y a pesar de las críticas generadas por el nivel de tradicionalismo en los procesos de enseñanza, ahora son las universidades quienes tienen el reto de liderar los modelos educativos a través de TIC y promover la formación de nuevas generaciones innovadoras, responsables de atender a las necesidades de la llamada sociedad del conocimiento. Por esta razón cabe señalar, conocer los efectos que trae consigo el uso de las TRIC en la formación de Licenciados, es un aspecto fundamental para determinar los aspectos que deben mejorarse desde diferentes aristas en todo el entorno universitario. Pues no cae duda que, su incorporación es necesaria para preparar a los ciudadanos en la fuerza laboral del futuro y facilitar a los estudiantes a aprender y a los maestros a enseñar de manera efectiva (Leung et al, 2005).

Marcillo (2016), asegura que las instituciones académicas de educación superior a través del uso de la tecnología, ofrecen experiencias de aprendizaje colaborativo a aquellos alumnos que no puedan viajar, lo que puede significar un impacto en el acceso a la educación y la experiencia de calidad. Las reflexiones son amplias y variadas, los cambios en la práctica y el rediseño pedagógico, deben darse para permitir maximizar los resultados del uso de la tecnología en los procesos educativos, aunque ello implique cambiar el enfoque pedagógico y las estrategias para administrar la clase con el propósito de mejorar el aprendizaje Bustos et al (2011). Por ello, vale la pena señalar que la presencia de la tecnología para la formación de Licenciados en la UPTC, no ha sido un proceso rápido como tampoco simple, con cuanto la graduación de profesionales de la educación en Colombia, es un camino exigente que requiere la aprobación de un número elevado de horas de práctica en las escuelas, que conlleven a determinar las competencias pedagógicas, didácticas y cognitivas que su título certifica; lo que deja entre dicho el uso masivo de las TIC para su formación en la Universidad, ya que no se conoce de modelos potencialmente utilizados en estás prácticas de formación específicas, además reconocidas y avalados por el Ministerio de Educación.

\section{METODOLOGÍA}

El enfoque de investigación en la cual se enmarca el desarrollo metodológico del estudio realizado es cuantitativo, que tradicionalmente se ha venido aplicando con éxito en investigaciones de tipo descriptivo, el cual exige la validez de la generalidad en los resultados y las conclusiones. Así, para alcanzar el principal propósito del estudio, en determinar los retos y oportunidades de incorporar las TIC en el programa de la Licenciatura, se realizó la categorización de los artículos consultados que argumenten el uso de las TIC en escenarios de educación superior, lo cual provee un soporte documental y científico a las características del tema que se aborda. Se pretende con los datos obtenidos a través de encuestas y observaciones realizadas, describir los efectos de la inclusión tecnológica en la calidad y la toma de decisiones frente al modelo educativo desarrollado para la formación de los estudiantes.

De esta manera, el desarrollo metodológico llevado a cabo se enmarcó en tres etapas fundamentales: 1. Determinación de un escenario educativo en donde el uso de las TIC fuera el protagonista; 2. Identificación de las particularidades del escenario en el cual se desarrolla la formación de los licenciados en educación Básica de la UPTC y 3. EI análisis de los efectos de incluir las TIC en la modalidad a distancia tradicional en la UPTC. De esta forma, se dio inicio a la revisión bibliográfica que permitiera la identificación de experiencias virtuales para la enseñanza en el nivel superior. En la primera etapa se realizó la selección de artículos producto de estudios científicos, así como de reflexiones académicas en torno al aprovechamiento de la 
tecnología en la educación. Los criterios de selección obedecen específicamente a que fueran escritos publicados en repositorios y bases de datos de reconocida trayectoria académica, tales como Scielo, Dialnet, Scopus y ScienceResearch, además del índice de citación y el autor de la publicación, que fuese una autoridad en el tema expuesto. La fecha de publicación es un criterio importante a tener en cuenta, sin embargo existen experiencias y escenarios en los cuales es valioso rescatar algunos postulados frente a la necesidad y la transformación que sufren los procesos de enseñanza cuando se utilizan medios tecnológicos; a pesar que en la actualidad dichos medios hayan evolucionado y cambiado la forma de comunicación de las personas. Los escritos en idioma inglés, así como las recomendaciones de expertos en el tema, hacen que su referenciación sea clave para los argumentos epistemológicos y científicos de la presente investigación.

En la fase 2 intervinieron 34 profesores y 102 estudiantes del programa de Licenciatura, a quienes se aplicó una encuesta con preguntas cerradas y abiertas, para identificar la percepción frente a los efectos del proceso educativo cuando la tecnología cobra protagonismo; lo cual generó una etapa de descripción y análisis en la cual se contrastó con la información analizada desde las experiencias consultadas en otros escenarios y los resultados obtenidos de los instrumentos aplicados a la población objeto de observación. Partiendo de la investigación documental, de las etapas de observación y análisis desarrolladas, se obtienen los resultados y las conclusiones que permiten contextualizar el escenario de la educación impartida en la UPTC con apoyo de la tecnología, dirigida a la población de Licenciados en Básica.

\section{RESULTADOS}

Los estudios muestran la importancia de la utilización y adaptación de la tecnología al servicio de estilos de aprendizaje diversos y sugieren que ésta por sí sola no constituye un factor de innovación, por cuanto el punto central está en posicionarla por encima de las realidades de los contextos educativos con particularidades distintas, en donde la aplicación dentro de la didáctica y el aprendizaje en el aula, debe ser más fuerte que su instrumentación en el modelo educativo.

Con base en los elementos conceptuales descritos y los instrumentos aplicados a la población objeto, se infiere que al ser las TIC un elemento importante para generar cambios en el modelo educativo; se ha dejado olvidada la necesidad de formar a los docentes, aun cuando se argumenta que la tecnología es una parte esencial para el desarrollo del aprendizaje, también es cierto que, el rol que desempeña el docente en el proceso educativo es fundamental, por cuanto, es quien potencializa la generación de conocimiento a través de estrategias pedagógicas en beneficio de las necesidades educativas del alumno, asunto que no se trata en ninguno de los planteamientos expuestos. Ortega y Casillas (2014) sostienen que se reconoce la importancia de la incursión de las TIC en los procesos de educación universitaria y coinciden además en el rol protagónico que desempeña el estudiante, cuando el aprendizaje surge a través de la mediación de herramientas tecnológicas.

El impacto de las TIC en los procesos de la educación dentro de la universidad, se enmarca en determinar sus efectos en la mejora del aprendizaje, este punto ha sido el eje central para vislumbrar los resultados de este apartado, donde se identifican las reflexiones de la incursión de las TIC propiamente para el desarrollo del proceso de aprendizaje en los estudiantes de Licenciatura en Básica de la UPTC. Así, en la Figura 2 se muestra la aprobación de la gran mayoría de la población, en el uso de la tecnología para el desarrollo del programa. Por tanto, se infiere que se considera adecuado el aprovechamiento de herramientas digitales en la metodología a distancia tradicional recibida desde hace varias décadas.

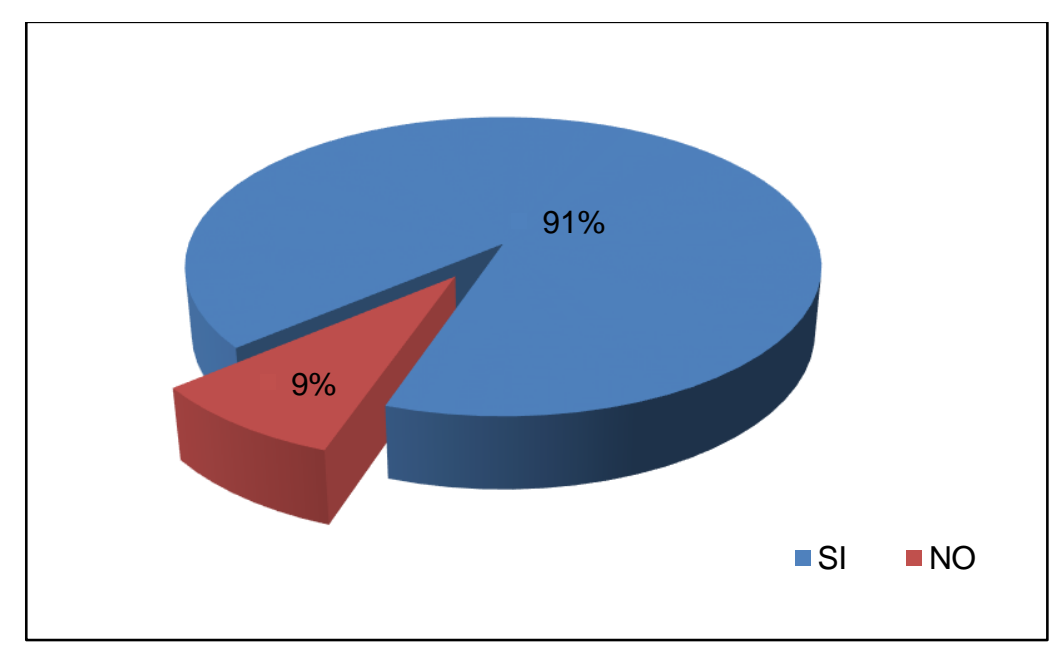

Fig. 2: Percepción del uso de la Tecnología en el programa LEB 
Frente a las ventajas del trabajo mediado por las TIC, en el proceso de formación a distancia del programa de LEB (Figura 3), se destacan aspectos como disponibilidad de recursos, facilidad en la información, acceso a los recursos desde lugares remotos y otros destacan la importancia como el ambiente de trabajo y el proceso de comunicación.

De los datos analizados y su contraste con los postulados expuestos, se considera una influencia positiva el uso de las TIC dentro de las universidades, pues se resalta el cambio metodológico, pedagógico y el aprovechamiento de materiales educativos a partir de la utilización de la tecnología en los procesos de mediación educativa. Esto conlleva a determinar aprendizajes diversos, orientados a un cambio de la educación superior en la universidad, que responda a las exigencias de la sociedad actual, producto de los avances acelerados de la tecnología y su campo de acción en todos los escenarios del mundo moderno. Así, el aprendizaje mediado por las TIC, vislumbra el nacimiento de un nuevo modelo de formación para Licenciados en Educación Básica, que den sentido a los cambios de la interacción educativa entre estudiantes, docentes y materiales de estudio; lo cual propicia el desarrollo de estructuras de aprendizaje diversas a partir de las necesidades e intereses particulares de formación como individuo y como comunidad. En consecuencia, el desarrollo de un modelo educativo donde la tecnología hace presencia de manera efectiva genera una transformación institucional orientada a responder a los retos que demanda el acceso a la información a partir de la sociedad del conocimiento.

La incidencia de la tecnología en el aprendizaje, ha fomentado no solo el desarrollo de metodologías innovadoras para la comunicación y la pedagogía; además el acceso a la educación de grupos heterogéneos que conlleva a la importancia de cambiar los enfoques de trabajo tradicional y el diseño, desarrollo y aprovechamiento de materiales de estudio que satisfagan las necesidades de respuesta a la información que se busca alcanzar. En tanto la educación mediada por TIC, específicamente en el Programa de Licenciatura en Educación Básica, facilita el acceso a la información rompiendo las barrearas de espacio y tiempo, y a su vez fomenta el mejoramiento del trabajo colaborativo, del aprendizaje individualizado, y la creación de nuevos sistemas de evaluación continua, que involucran la medición de los propósitos de formación, como a la vez la efectividad de la metodología desarrollada para ello.

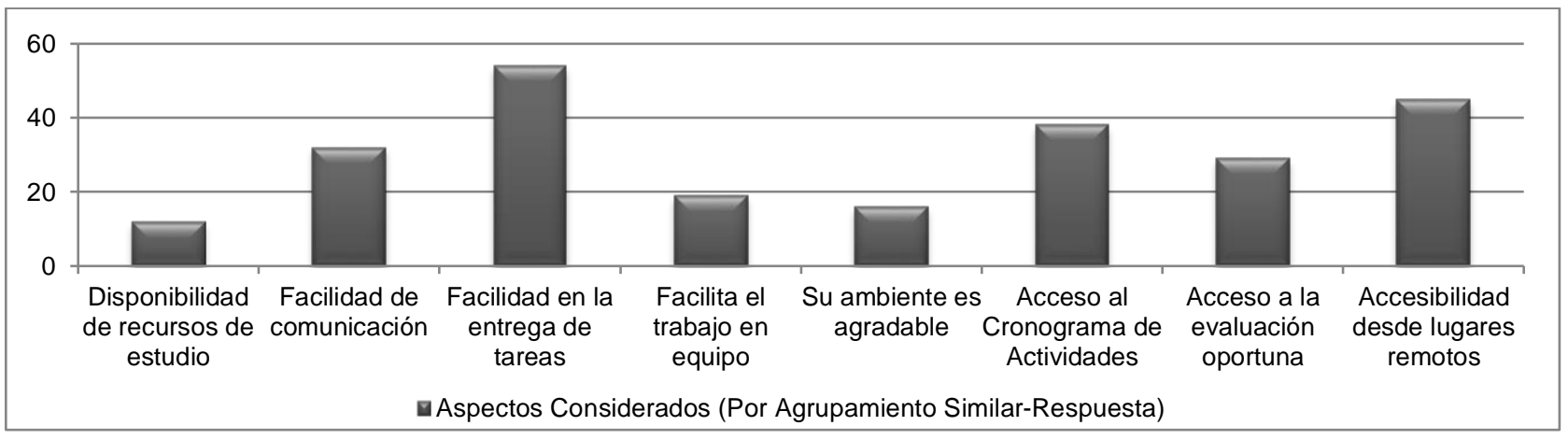

Fig 3: Percepción del uso de la Tecnología en el programa LEB

Ya lo afirman Facundo (2002) y Silvio (2000), en los resultados de sus investigaciones; que, llevar la tecnología a las aulas incrementa el reto de las universidades por buscar nuevos modelos educativos que comprometan la formación integral de los estudiantes, para que sean capaces de afrontar con competitividad las exigencias de la llamada sociedad del conocimiento, en donde las habilidades en el manejo y procesamiento de los datos que se transforman en información, mediante la utilización de los medios de comunicación electrónica, sean la mejor herramienta para aprovechar los conocimientos disciplinares que se adquieren durante el proceso de interacción con el programa educativo. Es así como, son las instituciones de educación superior las llamadas a liderar este cambio de paradigma entre la tradicionalidad de la enseñanza y el aprovechamiento de las TIC para mejorar los procesos comunicacionales y de interactividad proyectando cambios significativos en la educación superior con modalidad virtual. La figura 4 muestra la percepción de la población del programa frente al apoyo de los procesos de formación en la Educación a Distancia tradicional.

Por tanto, el éxito del aprovechamiento de las TIC en el proceso de aprendizaje dentro de la Licenciatura en Educación Básica, llevaba a cabo en la UPTC, no depende exclusivamente de la infraestructura tecnológica con la que se cuenta; lo que realmente hace la diferencia entre la tradicionalidad del aprendizaje y la formación mediada por tecnología, es el rol que desempeña el estudiante y el docente frente a la educación, la estrategia pedagógica implementada para alcanzar la interacción eficiente entre el estudiante y las fuentes de consulta, como también las oportunidades de mejoramiento continuo que se evalúan con el desarrollo de escenarios que se acerquen a la realidad de contexto en el cual se evidencia la educación recibida. 


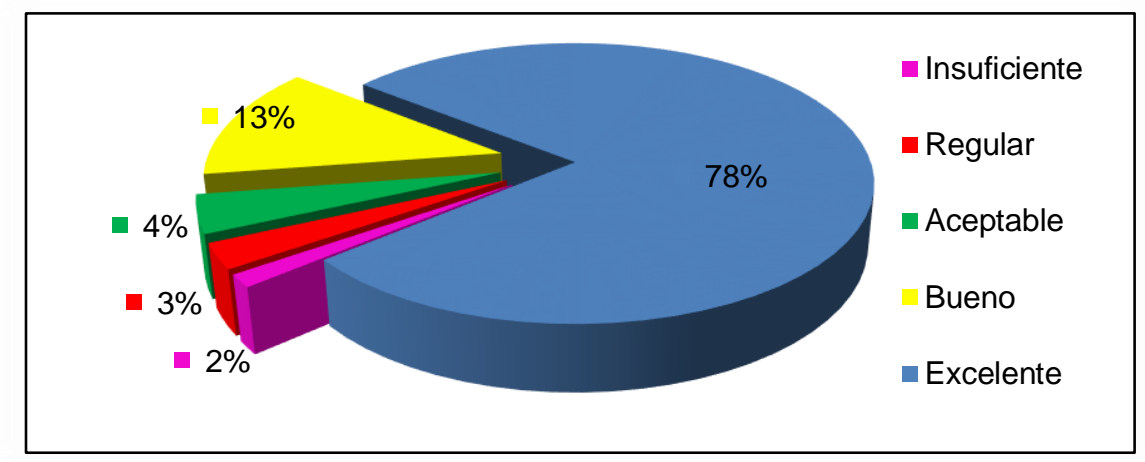

Fig.4: Percepción del uso de la Tecnología en el programa LEB

Es muy común que se despierten los debates académicos entorno a las ventajas, oportunidades y retos que trae la presencia de la tecnología en los procesos educativos, donde se han configurado escenarios ideales para trasformar la tradicionalidad del aprendizaje, dejando de manifiesto la necesidad de apoyar el desarrollo pedagógico en la universidad, con herramientas digitales que faciliten la comunicación. Sin embargo, estos aspectos conllevan a identificar la importancia de transformar los procesos educativos, donde la presencia de la tecnología abre horizontes para el mejoramiento y la continuidad de la calidad en la universidad. Se vislumbra el rompimiento de esquemas de aprendizaje individual, fortaleciendo la colaboración, la articulación de saberes y el desarrollo de habilidades comunicacionales que facilitan el aprendizaje. En este sentido y atendiendo a las tesis expuestas, la presencia de la tecnología en los procesos de aprendizaje en la UPTC, es mucha; el diseño de entornos formativos flexibles en tiempo y espacio, con actividades de aprendizaje que favorezcan el desarrollo de un proceso cognitivo real, además del cambio a los tradicionales roles dentro del ejercicio pedagógico, como la estrategia para responder con los retos de la actual sociedad.

El estudio da cuenta de los beneficios de las TIC en los procesos educativos llevados a cabo al interior de las universidades. Sin embargo, en contextos y realidades distintas, no se responde a las condiciones que se abordan, con los elementos propios y particulares que busquen atender a las exigencias de la sociedad, haciendo cambio de roles en el ejercicio de aprendizaje mediado por la tecnología, sin desconocer los propósitos de aprendizaje que encaminan el proceso educativo específico, que se adelanta en la disciplina 0 campo de formación. Los argumentos presentados, producto de diversos estudios, son claves para considerar distintos elementos que en el campo de la pedagogía y la enseñanza son esenciales para alcanzar los objetivos de formación enmarcados en los planes de estudio, pues si bien se ha dicho a lo largo de los resultados de las investigaciones de la importancia de usar las TIC en los procesos de aprendizaje, también se ha dejado de lado, establecer las realidades educativas y sociales en los cuales se desarrollarían estos planeamientos, desde la propias limitaciones de acceso o brecha tecnoeducativa, que enmarcan las generaciones antes y después de la era digital.

Los retos y las oportunidades del proceso de utilización de las TIC en el campo del aprendizaje, en particular la Licenciatura en Educación Básica de la UPTC, permiten reinventar el modelo educativo, y centrar su atención en el estudiante, por cuanto el deseo de aprender, el aprovechamiento de las herramientas y la motivación permanente de acceder al conocimiento sigue estando visible en el ser y no en la tecnología.

\section{CONCLUSIONES}

1. Las TIC cobran un rol importante para el desarrollo de las estrategias docentes en el programa de Licenciatura en Educación Básica; las cuales permiten la visibilización de la flexibilidad curricular de la UPTC y el desarrollo de un modelo educativo combinado en donde se apoyen los procesos tradicionales de la educación a distancia con el uso adecuado de herramientas digitales para la comunicación e interacción.

2. El aprovechamiento de las TIC en los procesos de formación han determinado que del rol que asuman los estudiantes depende el éxito del ejercicio académico; así como a la vez la capacitación y actualización a docentes, que les permitan responder a las necesidades actuales de mediación pedagógica en ambientes educativos donde se hace cada vez más necesaria la interacción digital. La pandemia por Covid19, ha resaltado en gran medida, la necesidad que se tiene de la presencia de las TIC en los procesos educativos.

3. Las TRIC en los procesos educativos, han de estar articuladas con las estrategias pedagógicas, metodológicas y comunicacionales que a partir del ejercicio docente se haga en el proceso formativo; así el eje educativo central se enmarca en los objetivos de aprendizaje que conlleve a mantener la calidad de la educación superior, al mismo tiempo que enmarca la transformación de un modelo educativo activo y participativo en el cual la era de la digitalización se pone al servicio de la pedagogía. 
4. Con el aprovechamiento de las TIC en el modelo educativo de la formación de licenciados en la UPTC, se hace una resignificación de su concepto y se eleva su importancia a la construcción de diversas formas de acceder y transformar el aprendizaje; en la búsqueda del crecimiento personal y colectivo para la adquisición de nuevos saberes dentro de una disciplina; en la cual las TRIC son un elemento importante para la adquisición de conocimiento significativo para su ejercicio profesional al interior de la escuela.

5. Los debates epistemológicos y las inferencias que se obtienen en los resultados del estudio al interior del programa de Educación Básica, dejan entrever la necesidad de promover trasformaciones más profundas en el modelo, la metodología y las estrategias de aprendizaje que hasta hace algún tiempo son desarrolladas sin mayor cuestionamiento en las instituciones educativas. Las universidades han sido temerosas en afrontar con decisión el uso de la tecnología dentro de sus prácticas docentes, sin embargo la presencia de la pandemia Covid19, ha dejado al descubierto los vacíos del tema en sus escenarios de formación, lo cual ha despertado números retos que conllevan a cambios profundos en las tradicionales formas de gestionar el conocimiento.

6. Los beneficios que traen las TIC en la educación, han dejado al descubierto la imperiosa necesidad de capacitación y actualización docente, para enfrentar con exigencia las nuevas circunstancias que ha traído el Covid19 a los procesos de formación universitarios. El evidente protagonismo de la tecnología en la educación, genera cambios significativos tanto en las estrategias utilizadas para la enseñanza, como en el modelo comunicacional para mantener la calidad del servicio educativo en las actuales circunstancias.

\section{REFERENCIAS}

Aranguiz, M. S. y Quintana, M. G. ICT resources to improve learning in higher education. International Journal of Knowledge Society Research (IJKSR), 7(4), 1-11. (2016).

Bustos, H. A., Delgado, M. A., y Pedraja, R. L. Inclusion strategy for mobile technology in the classroom: experience at the Universidad de Tarapacá. Ingeniare. 10.4067/S0718-33052011000100003. Revista chilena de ingeniería, 19 (1), 1925 (2011).

Colina, L. C., Las TIC en los procesos de enseñanza-aprendizaje en la educación a distancia. Laurus, 14(28), 295-314. (2008).

Coll, C., Aprender y enseñar con las TIC: expectativas, realidad potencialidades. Boletín de la Institución Libre de Enseñanza, Boletín de la Institución Libre de Enseñanza, 72, 17-40, (2008).

Dave, DD. Un estudio analítico del papel de las TIC en la educación superior. doi.org/10.1956/jge.v15i1. Journal of Global Economy , 15 (1 (Especial), 56-61. (2019).

Facundo, Á. Educación virtual en América Latina y el Caribe: características y tendencias. Bogotá: Universidad de El Libertador. (2002).

Garrote, D., Jiménez, S., Gómez, I. M. Problemas Derivados del Uso de Internet y el Teléfono Móvil en Estudiantes Universitarios. dx.doi.org/10.4067/S0718-50062018000200099. Formación Universitaria. ISSN-e 0718-5006. Vol. 11 № 2. (2018).

Ghavifekr, S., Razak, A. Z., y cuatro autores más. ICT integration in education: Incorporation for teaching and learning improvement. Malaysian Online Journal of Educational Technology, 2(2), 24-45. (2014).

Herrera, A. M. Una mirada reflexiva sobre las TIC en Educación Superior. Revista electrónica de investigación educativa versión On-line ISSN 1607-4041 (2015).

Kinuthia, W. y Dagada, R. E-learning incorporation: an exploratory study of three South African higher education institutions. International Journal on E-learning, 7(4), 623-639. (2008).

Leung, K., Watters, J., y Ginns, I. Enhancing teachers' incorporation of ICT in classroom teaching. (2005).

Marcillo, M. y Desilus, B. Collaborative Online International Learning Experience in Practice Opportunities and Challenges. 10.4067/S0718-27242016000100005 Journal of technology management \& innovation, 11(1), 30-35. (2016).

Martínez, O. M., Steffens, E. J., Ojeda, D. C., Hernández, H. Estrategias Pedagógicas Aplicadas a la Educación con Mediación Virtual para la Generación del Conocimiento Global. dx.doi.org/10.4067/S0718-50062018000500011. Formación Universitaria Vol. 11 № 5. (2018).

Mendoza, H. H., Burbano, V. M., Valdivieso, M. A. El Rol del Docente de Matemáticas en Educación Virtual Universitaria. Un Estudio en la Universidad Pedagógica y Tecnológica de Colombia. dx.doi.org/10.4067/S0718-50062019000500051 (2019). Formación Universitaria Vol. 12 № 5 (2019).

Ortega, M. y Casillas, M. A. Háblame de TIC. Tecnología digital en la Educación Superior. (2014).

Remache, A. y Belarbi, A. Adaptar las TIC en la educación superior en el mundo en desarrollo: influir en la dinámica. Revista Internacional de Política Económica en Economías Emergentes, ISSN: 1752-0460. (2019).

Salinas, J. Innovación docente y uso de las TIC en la enseñanza universitaria. International Journal of Educational Technology in Higher Education (ETHE), 1(1). (2004). 
Sánchez, M., Añorve, JR y Alarcón, G. Las Tic en la educación superior, innovaciones y desafíos / Las TIC en educación superior, innovaciones y desafíos. RICSH Revista lberoamericana de las Ciencias Sociales y Humanísticas, ISSN 239579726 (12), 299-316. (2017).

Saviani, D. Pedagogy: the space for education at the university. 10.1590/S0100-15742007000100006. Cuadernos de Pesquisa, 37(130), 99-134. (2007).

Silvio, J. La Virtualización de la Universidad:¿ Cómo transformar la educación superior con la tecnología?. Caracas, (2000).

Sointu, E. T., Valtonen, T. y cinco autores más. Teachers as users of ICT from the student perspective in highereducation flipped classroom classes. En seminario. net Vol. 15, No. 1, pp. 1-15. (2019).

Valentín, A., Mateos, P. M., González, M. M., Pérez, L., López, E., y García, I. Motivation and learning strategies in the use of ICTs among university students. Computers and Education, 61, 52-58. (2013). 